\title{
Rectificador monofásico con corrección del factor de potencia usando un convertidor Boost
}

\section{Power factor correction over single-phase rectifiers using a Boost converter}

\section{Lenny Andrés Hernández Fonseca}

Ingeniero electrónico. Investigador de la Universidad Pedagógica y Tecnológica de Colombia. Sogamoso, Colombia. Contacto:landres87@hotmail.com

\section{Diego Ricardo Gómez León}

Ingeniero electrónico. Investigador de la Universidad Pedagógica y Tecnológica de Colombia. Sogamoso, Colombia. Contacto: richardgole@hotmail.com

\section{Oscar Mauricio Hernández Gómez}

Ingeniero electrónico. Docente e Investigador de la Universidad Pedagógica y Tecnológica de Colombia. Sogamoso, Colombia.

Contacto: oscarmauricio.hernandez@uptc.edu.co

Fecha de recepción: 31 de agosto de 2011

Fecha de aceptación: 17 de abril de 2012

Clasificación del artículo: Investigación

Palabras clave: calidad de la potencia, convertidor de potencia AC-DC, convertidor de potencia DC-DC, factor de potencia, sistemas de potencia.

Key words: power quality, AC-DC power conversion, DC-DC power conversion, power factor, power system. 


\section{RESUMEN}

En este artículo se analiza el modelo matemático y el diseño de controladores para el convertidor DC-DC tipo Boost, enfocado en la corrección del factor de potencia. El convertidor se diseñó a $24 \mathrm{~W}$ de potencia, se muestran las simulaciones en MATLAB y los resultados experimentales obtenidos del convertidor.

\section{INTRODUCCIÓN}

Con el incremento de los dispositivos electrónicos se ha aumentado el uso de rectificadores que, debido a sus características de funcionamiento, hacen que la corriente de entrada sea no sinusoidal, causando una alta distorsión armónica en corriente, reduciendo con esto el factor de potencia en la entrada del rectificador [1].

La mayoría de los electrodomésticos presentan un rectificador de voltaje en la entrada de la alimentación que disminuye el factor de potencia, al analizar esta situación se puede observar que si se maneja un bajo factor de potencia se presentan una gran cantidad de problemas, tanto para el usuario como para la empresa prestadora del servicio de energía, entre estos encontramos: fuertes caídas de tensión, pérdidas y aumento de la temperatura de los conductores, lo cual disminuye la vida de su aislamiento, elevadas caídas de tensión y baja regulación de voltaje, lo cual puede afectar la estabilidad de la red eléctrica; mayor inversión en los equipos de generación, ya que su capacidad en KVA debe ser mayor para poder entregar esa energía reactiva adicional [2], entre otros. Por tal motivo, se deben tomar medidas correctivas que ayuden a evitar y controlar estos problemas para que los dispositivos electrónicos que se estén manipulando puedan funcionar de manera adecuada y respondan a las exigencias que se deseen.

\section{ABSTRACT}

This paper shows a mathematical model and corresponding controller design of a $24 \mathrm{~W}$ DC-DC Boost converter used for power factor correction. Modeling and simulations are shown (using Matlab) together with experimental results.

Actualmente, en la unión americana y europea existen estándares para la regulación de armónicos en la línea de alimentación generadas por convertidores de potencia, infortunadamente la contaminación armónica en el ámbito domiciliario de países como Colombia es indiscriminada. Por tanto, es necesaria la investigación de nuevos sistemas de alimentación que sean más eficientes y corrijan el factor de potencia cumpliendo con los estándares internacionales [3].

En la actualidad, varios autores han escrito sobre la corrección del factor de potencia haciendo uso de convertidores. Singh y otros [4] hacen un resumen acerca de los diferentes tipos de convertidores y sus topologías para la corrección del factor de potencia.

Tahami, et al [5] ilustran el modelado matematico del convertidor boost en modo de conducion mixta, es decir, en modo de conducción continua y en conducción discontinua.

Zhou [6] muestra el diseño de un circuito para la correccion del factor de potencia con control variable de la histeresis para una fuente suichada de potencia.

Así entonces, en este artículo se muestra la forma de implementar un rectificador monofásico con corrección del factor de potencia haciendo uso de un convertidor DC-DC tipo Boost, para 


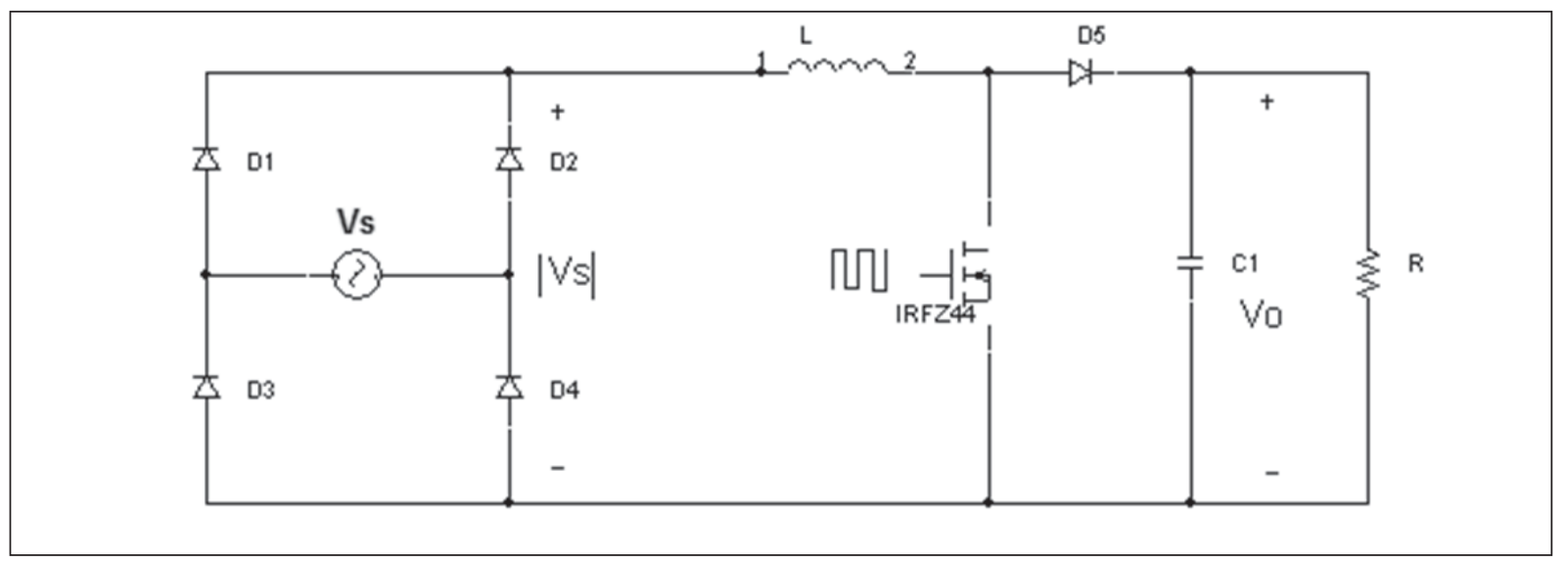

Figura 1. Circuito convertidor Boost.

Fuente: elaboración propia

lo cual se consultan los diferentes avances tecnológicos y teoría relacionada con los métodos para la corrección del factor de potencia, posteriormente se desarrollan los modelos matemático y conmutado del convertidor, así como su controlador; y se realizan las simulaciones de los modelos por medio de MATLAB para su posterior implementación.

\section{METODOLOGÍA}

\subsection{Modelo matemático del convertidor}

En el modelo matemático [7] que se va a desarrollar a continuación se considera inicialmente que el convertidor boost, mostrado en la figura 1 , trabaja en modo conducción continua (MCC), es decir que la corriente en la bobina no se hace cero en ningún instante de tiempo. Esta situación se logra cumpliendo la relación mostrada en la ecuación (1), donde $L$ representa la inductancia, $f$ la frecuencia de conmutación, $R$ la resistencia de carga y $D$ el ciclo útil del convertidor.

$$
\frac{2 L f}{R}>D(1-D)^{2}
$$

Para describir el comportamiento matemático del convertidor, se utiliza la técnica de espacio de estados que define dos posiciones de funcionamiento basados en la operación de conmutación del MOSFET (figura 1), enseguida se definen dos variables de estado en el sistema, corriente en el inductor y voltaje en el capacitor el cual será el mismo voltaje de salida. Los circuitos equivalentes para ambas condiciones se observan en las figura 2 y figura 3 respectivamente.

\subsubsection{Ecuaciones de estado}

En la figura 2 se observa el circuito equivalente del convertidor Boost cuando está activo el MOSFET con una resistencia de encendido $R_{o n}$ y el diodo está apagado, teniendo en cuenta $R_{l}$

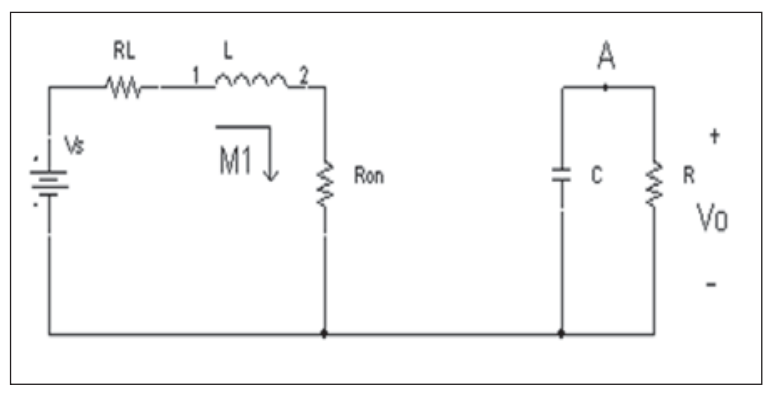

Figura 2. Circuito equivalente con el MOSFET activo. Fuente: elaboración propia 


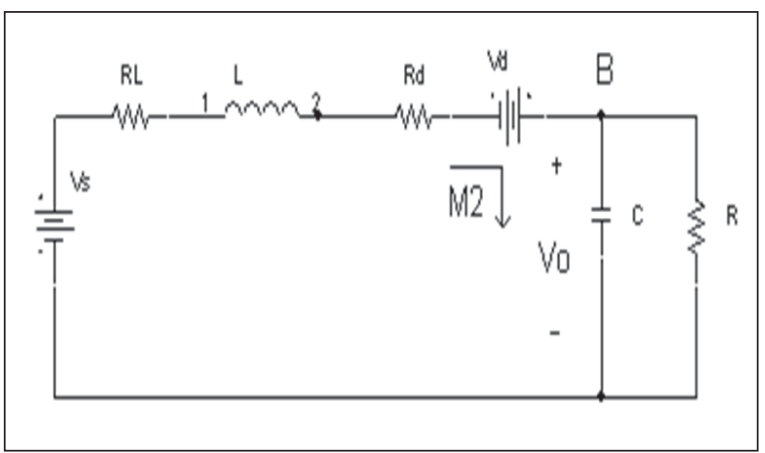

Figura 3. Circuito equivalente con el diodo activo

Fuente: elaboración propia

como la resistencia de la inductancia, $L$ valor de inductancia, $C$ el valor del condensador, $v_{s}$ el voltaje de alimentación, $v_{o}$ el voltaje de salida y $R$ la resistencia de carga nominal. Las ecuaciones de estado para el circuito de la figura 2 se presentan en la ecuación (2) y la ecuación (3).

$$
\begin{gathered}
\frac{d i_{l}(t)}{d t}=\frac{-i_{l}(t) R_{L}+\mathrm{v}_{\mathrm{s}}-i_{l}(t) R_{o n}}{L} \\
\frac{d v_{c}(t)}{d t}=\frac{-v_{c}(t)}{C R}
\end{gathered}
$$

En la figura 3 se observa el circuito equivalente del convertidor Boost cuando está activo el diodo con una resistencia interna $R_{d}$ y un voltaje de activación $V_{d}$, estando el MOSFET apagado, sus ecuaciones de estado se presentan en la ecuación (4) y (5).

$$
\begin{gathered}
\frac{d i_{l}(t)}{d t}=\frac{v_{\mathrm{s}}-i_{l}(t) R_{l}-v_{d}-v_{c}(t)-i_{l}(t) R_{d}}{L} \\
\frac{d v_{c}(t)}{d t}=\frac{i_{l}(t)}{C}-\frac{v_{c}(t)}{C R}
\end{gathered}
$$

Las ecuaciones (2) a (5) son expresadas en un solo espacio de estados que representa el comportamiento del convertidor de gran señal expresado en ecuación (6).

$$
\begin{aligned}
& {\left[\begin{array}{l}
\hat{i}_{L}(t) \\
\hat{v}_{c}(t)
\end{array}\right]=\left[\begin{array}{cc}
\frac{-R_{l}-R_{O N} D-R_{d} D^{\prime}}{L} & \frac{-D^{\prime}}{L} \\
\frac{D^{\prime}}{C} & \frac{-1}{C R}
\end{array}\right] *\left[\begin{array}{c}
i_{l}(t) \\
v_{c}(t)
\end{array}\right]+} \\
& {\left[\begin{array}{cc}
\frac{1}{L} & 0 \\
0 & 0
\end{array}\right] *\left[\begin{array}{c}
\mathrm{v}_{\mathrm{s}} \\
0
\end{array}\right]+\left[\begin{array}{c}
\frac{\left(-R_{O N}+R_{d}\right) i_{l}(t)+v_{c}(t)+v_{d}}{L} \\
-\frac{i_{l}(t)}{c}
\end{array}\right] * d(t)}
\end{aligned}
$$

\subsubsection{Perturbación y linealización}

Con el espacio de estados de la ecuación (6) se debe perturbar y linealizar el sistema alrededor del punto de operación, para lo cual se tiene en cuenta que las variables se pueden representar como una suma de sus valores estáticos o DC, representados por letras mayúscula y dinámicos o de variación AC, representados con letras minúsculas. Realizando un tratamiento matemático se agrupan los términos AC, DC y de segundo orden. Tomando los términos de las variaciones AC se expresa la corriente en el inductor como se muestra en la ecuación (7).

$$
\begin{aligned}
& \frac{L d(\hat{i}(t))}{d t}=\left(-R_{t}-R_{O N} D-R_{d} D^{\prime}\right) \hat{i}(t)-D^{\prime} v_{c}(t)+\hat{\mathrm{v}}_{\mathrm{s}}+(7) \\
& \left(-R_{O N}+R_{d}\right) \mathrm{I} \hat{d}(t)+V_{c} \hat{d}(t)
\end{aligned}
$$

De igual manera se agruparon las variaciones para la variable de estado del voltaje en el condensador como se aprecia en la ecuación (8).

$$
\frac{C d v_{c}(t)}{d t}=D^{\prime} \hat{i}_{l}(t)-\frac{\hat{v}_{c}(t)}{R}-I_{l} \hat{d}(t)
$$

\subsubsection{Función de transferencia}

Posteriormente, se debe hallar la función de transferencia [7] del sistema, para lo cual se analiza el circuito del modelo equivalente de pequeña señal mostrado en la figura 4, el cual fue deducido con la ecuación (7) y ecuación (8).

Debido a que el convertidor contiene dos entradas independientes, las cuales son el control del 


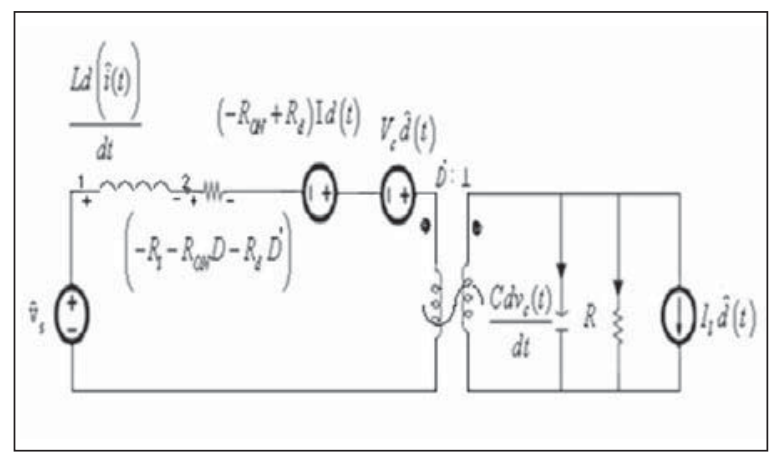

Figura 4. Modelo equivalente de pequeña señal del convertidor boost.

Fuente: elaboración propia

PWM y la línea de entrada de voltaje AC, las variaciones del voltaje de salida AC serán expresadas como una superposición de las dos entradas para facilitar el análisis en la ecuación (9).

$$
V_{\mathrm{o}}=\mathrm{G}_{\mathrm{vd}} \hat{d}(s)+\mathrm{G}_{\mathrm{vg}} \hat{v}_{g}(s)
$$

Se toma la ecuación (9) y se deduce la función de transferencia del voltaje de salida con respecto a la señal de control y el voltaje de salida con respecto al voltaje de entrada como se expresa en la ecuación (10).

$$
\left.\left.\mathrm{G}_{\mathrm{vd}}(s)=\frac{\hat{v}_{c}(s)}{\hat{d}(s)}\right]_{\mathrm{v}_{\mathrm{s}}=0} \mathrm{G}_{\mathrm{vg}}(s)=\frac{\hat{v}_{c}(s)}{\hat{v}_{s}(s)}\right]_{\hat{d}(s)=0}
$$

Para la primera función de transferencia se elimina la fuente Vs y se utiliza la relación de transformación del modelo $D^{\prime}: 1$ para redibujar el circuito observado desde el lado de la carga en el dominio de S, se realiza el análisis y se obtiene la función de transferencia en la ecuación (11).

$$
\frac{\hat{v}_{c}(s)}{\hat{d}(s)}=\frac{\frac{V_{c}}{C}\left(-\frac{s}{R D^{\prime}}+\frac{\alpha_{1}}{L R D^{\prime}}+\frac{\alpha_{2}}{R L}-\frac{D^{\prime}}{L}\right)}{s^{2}+\frac{s}{C R}+\frac{s \alpha_{1}}{L}+\frac{\alpha_{1}}{R L C}+\frac{D^{\prime 2}}{L C}}
$$

Se realiza la sustitución mostrada en ecuación (12) y ecuación (13) para reducir términos en la ecuación (11).

$$
\begin{gathered}
\alpha_{1}=\left(R_{l}+R_{O N} D+R_{d} D^{\prime}\right) \\
\alpha_{2}=\left(R_{O N}-R_{d}\right)
\end{gathered}
$$

\subsection{Diseño del convertidor Boost}

El convertidor Boost se implementó utilizando los valores mostrados en la ecuación (14):

$$
\begin{aligned}
& \mathrm{V}_{\text {in }}=12 \mathrm{v} \text { rms } \quad \mathrm{V}_{\mathrm{o}}=24 \mathrm{v} \mathrm{DC} \\
& \mathrm{R}=24 \Omega \quad \mathrm{I}_{\text {carga }}=1 \mathrm{~A} \quad \mathrm{Ron}=0.3 \Omega \\
& \mathrm{Rd}=0.5 \Omega \quad \mathrm{Vd}=0.62 \mathrm{v} \mathrm{P}_{\mathrm{o}}=24 \mathrm{~W} \mathrm{R}_{l}=1.3 \Omega
\end{aligned}
$$

Se calcula el ciclo útil según en la ecuación (15), para cumplir con el voltaje de salida deseado.

$$
D=\frac{V_{o}-V_{i n}}{V_{o}}=\frac{24-12}{24}=0,5
$$

El valor de la inductancia es expresado por la ecuación (16).

$$
L=\frac{V_{i n} D}{F_{s} \Delta I}=81,4 \mu H
$$

El valor del condensador de salida será hallado por medio de la ecuación (17).

$$
C=\frac{2 * P_{o} \Delta t}{V_{o}^{2}-V_{\text {in }}^{2}}=6,23 m F
$$

\subsection{Diseño de controladores}

\subsubsection{Diagramas de Bode}

Para analizar la respuesta en frecuencia de la función de transferencia en la ecuación (11), se grafican los diagramas de bode de magnitud y fase como se observa en la figura 5 , donde se puede apreciar una frecuencia de corte de $4800 \mathrm{rad} / \mathrm{seg}$ y su margen de fase de 13.1 grados.

Posteriormente para hallar la segunda función de transferencia se hace un análisis similar, pero en este caso se elimina la señal y se analiza matemáticamente para obtener la función de transferencia mostrada en la ecuación (18). 


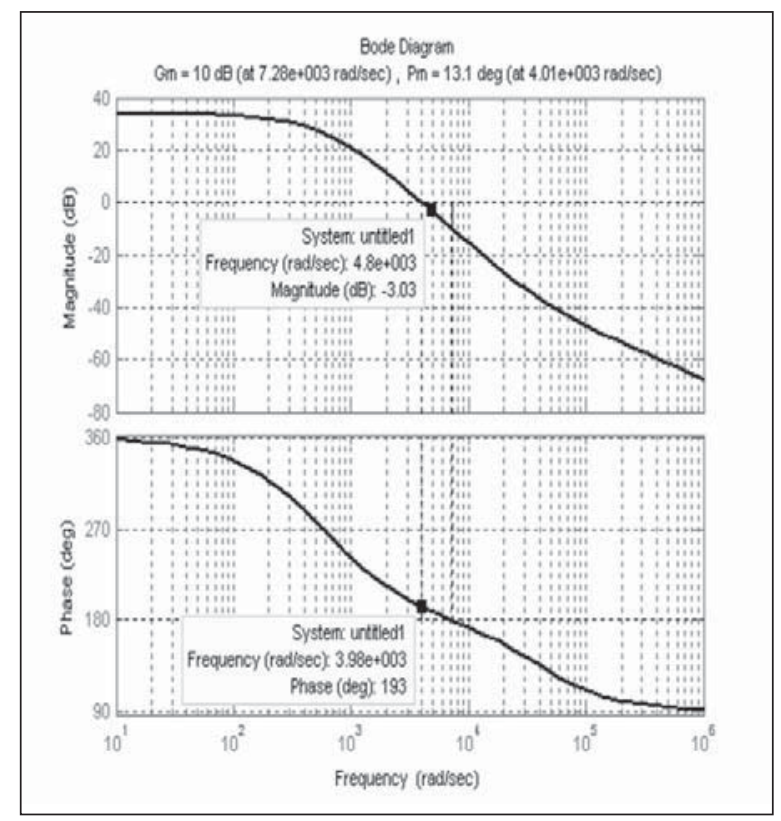

Figura 5. Diagrama de bode de la función de transferencia del voltaje de salida con respecto a la señal de control.

Fuente: elaboración propia

$$
\frac{\hat{v}_{c}(s)}{\hat{v}_{s}(s)}=\frac{\frac{D^{\prime}}{L C}}{s^{2}+\frac{s}{C R}+\frac{s \alpha_{1}}{L}+\frac{\alpha_{1}}{R L C}+\frac{D^{\prime 2}}{L C}}
$$

Para analizar la respuesta en frecuencia de la función de transferencia la ecuación (18), al igual que en la ecuación (11), se grafican los diagramas de bode de magnitud y fase como se observa en la figura 6 en donde se puede apreciar una frecuencia de corte de $691 \mathrm{rad} / \mathrm{seg}$ y un margen de fase de 99.5 grados.

La ecuación (19) representa la respuesta total del sistema en función de las dos entradas en el dominio de $\mathrm{S}$.

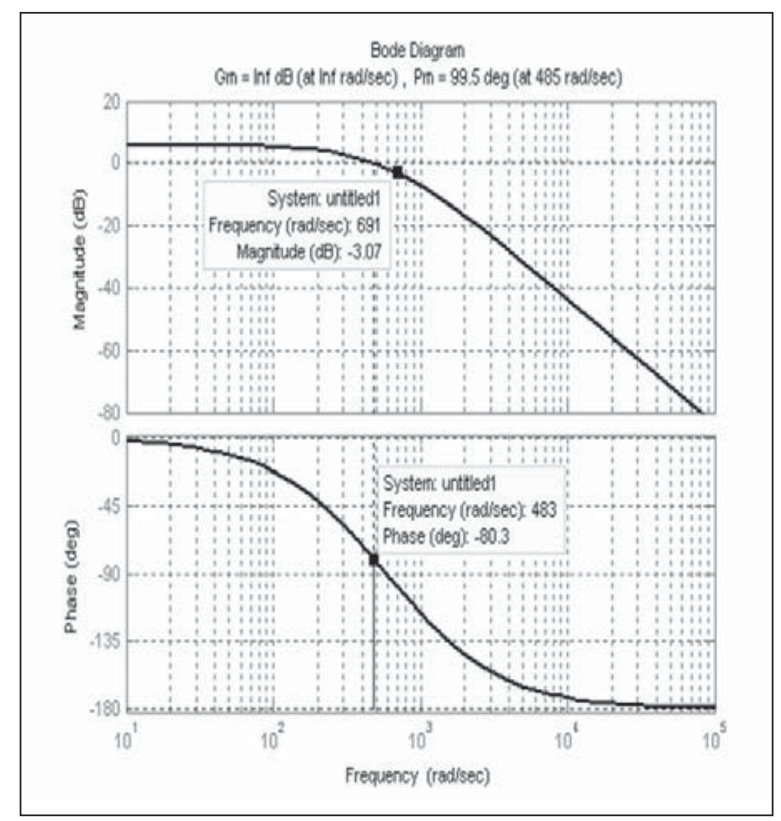

Figura 6. Diagramas de bode de la función de transferencia del voltaje de salida con respecto al voltaje de entrada

Fuente: elaboración propia

El control de un PFC [8]-[10] (Power Factor Correction) se basa, principalmente, en forzar la señal de corriente del generador a dibujar una trayectoria senoidal y regular el voltaje de salida para cumplir con la especificación de potencia requerida en la carga, por lo cual, es necesario tener dos lazos de control, uno de corriente y otro de voltaje.

El lazo interior de corriente manipula la forma de onda de la corriente $I_{L}$, utilizando la forma de onda del voltaje $\left|V_{s}\right|$, es decir, que la señal de referencia del controlador de corriente será una señal totalmente rectificada.

El lazo exterior de voltaje determina la amplitud de $I_{L}$ basado en la salida del voltaje realimentado. Si la corriente del inductor es insuficiente para

$$
\hat{v}_{c}(s)=\frac{\frac{V_{c}}{C}\left(-\frac{s}{R D^{\prime}}+\frac{\alpha_{1}}{L R D^{\prime}}+\frac{\alpha_{2}}{R L}-\frac{D^{\prime}}{L}\right) * \hat{d}(s)}{s^{2}+\frac{s}{C R}+\frac{s^{*} \alpha_{1}}{L}+\frac{\alpha_{1}}{R L C}+\frac{D^{\prime 2}}{L C}}+\frac{\frac{D^{\prime}}{L C} * \hat{v}_{s}(s)}{s^{2}+\frac{s}{C R}+\frac{s \alpha_{1}}{L}+\frac{\alpha_{1}}{R L C}+\frac{D^{\prime 2}}{L C}}
$$




\section{investigación}

alimentar la carga del PFC, la salida de voltaje bajará un poco del valor de referencia. Tomando la señal de voltaje de salida como señal de realimentación, el lazo de voltaje ajustará la amplitud de la corriente del inductor llevando el voltaje de salida al valor de referencia.

El lazo interior de corriente tiene un ancho de banda superior al lazo exterior de voltaje, esto se da debido a que es necesario que el controlador de corriente responda más rápido que el controlador de voltaje, para tener una corrección optima de la señal de corriente en el generador $I_{g}$.

\subsubsection{Diseño del controlador de corriente}

El controlador de corriente [10], [12] debe tener un polo en el origen, el cual le dará una respuesta más rápida al compensador. Con el polo de $G_{C I}(s)$ expresado en la ecuación (20) y el de la función de transferencia del convertidor, le adicionan -180 grados al lazo dándole un margen de estabilidad aceptable.

$$
G_{C I}(s)=\frac{K_{c}\left(1+\frac{s}{w_{z}}\right)}{s\left(1+\frac{s}{w_{p}}\right)}
$$

Se desea un margen de fase de $60^{\circ}$ para tener una respuesta suave del convertidor por tal motivo se asume un $\varphi_{\text {boost }}$ de $60^{\circ}$, como se analizó anteriormente en el diseño del lazo de corriente, el ancho de banda del controlador debe ser muy grande, por tal motivo se asume una frecuencia de corte $\left(F_{c i}\right)$ de $10 \mathrm{KHz}$, con estos valores se procederá al diseño del compensador PI mostrado en la ecuación (20), el término $K_{\text {boost }}$ indica la separación geométrica entre el polo y el cero necesaria para obtener el margen de fase como se expresa en la ecuación (21).

$$
K_{\text {boost }}=\tan \left(45+\frac{\varphi_{\text {boost }}}{2}\right)=3,732
$$

Con este valor se hallaran las frecuencias donde se ubicaran el cero en la ecuación (22) y el polo en la ecuación (23) del controlador respectivamente.

$$
\begin{aligned}
& f_{z}=\frac{f_{c i}}{K_{\text {boost }}}=2,676 \mathrm{kHz} \\
& f_{p}=f_{c i} K_{\text {boost }}=37,320 \mathrm{Khz}
\end{aligned}
$$

Con ayuda de la figura 6 se calcula el valor en $d b$ de ${ }_{z}^{\prime} \mid G_{v g}(s$ para hallar la ganancia del controlador $K_{c}$ mostrada en la ecuación (24).

$$
\begin{aligned}
& K_{c}=w_{z}\left|G_{v g}(s)\right|_{f c}=(16835,97)(14,64)=123242,09 \\
& W_{z}=16835,97 \quad w_{p}=234488,47 \quad K_{c}=123242,09
\end{aligned}
$$

En la ecuación (25) se representa la función de transferencia del controlador de corriente que será implementada en el convertidor para la corrección del factor de potencia.

$$
G_{C I}(s)=\frac{123242,09}{s} \frac{\left(1+\frac{s}{16835,97}\right)}{\left(1+\frac{s}{234488,47}\right)}
$$

\subsubsection{Diseño del controlador de voltaje}

En la ecuación (26) se presenta la función de transferencia del compensador de voltaje [8]-[10], la cual contiene un polo ubicado en la frecuencia de corte $f_{c v}$ que será menor a $15 \mathrm{hz}$ esto con el fin de atenuar las frecuencias de la red $(60 \mathrm{y} 120 \mathrm{~Hz})$.

$$
G_{c v}(s)=\frac{K_{v}}{1+\frac{s}{w_{c v}}}
$$

La función de transferencia a plena carga del convertidor tiene un polo a muy baja frecuencia, el cual introduce una fase de aproximadamente $90^{\circ}$ en atraso. El controlador de voltaje introducirá $45^{\circ}$ en atraso a la frecuencia de corte del lazo lo que proporcionará un margen de fase satisfactorio de $45^{\circ}$. Por definición [8] la función de transferencia del lazo tiene una magnitud igual a la uni- 
dad en la frecuencia de corte. Como se observa en la ecuación (27).

$$
\left|\frac{K_{v}}{1+\frac{s}{w_{c v}}} \frac{1}{2} \frac{v_{s}}{v_{d}} \frac{R}{1+s R c}\right|_{f c v}=1
$$

En la ecuación (28) se muestra la magnitud del controlador evaluada en $120 \mathrm{~Hz}$, que representa la inyección del segundo armónico al sistema debido al control.

$$
\left|\frac{K_{v}}{1+\frac{s}{w_{c v}}}\right|_{s \rightarrow j 2 \pi 120}=\frac{I_{L 2}}{V_{o 2}}
$$

$I_{L 2}$ es igual a $1,5 \%$ de la corriente de referencia y $V_{o 2}$ hace referencia al valor del voltaje pico de entrada rectificado.

$K_{v}$ y $w_{c v}$ son las dos variables desconocidas de manera que resolviendo la ecuación (27) y ecuación (28) se obtienen las constantes del controlador, así su función de transferencia se presenta en la ecuación (29).

$$
G_{c v}(s)=\frac{0,1812}{1+\frac{s}{62,62}}
$$

\subsection{Análisis del convertidor en lazo cerrado}

Después de haber obtenido las funciones de transferencia del sistema, se debe analizar su comportamiento en lazo cerrado; como el esquema de control de la figura 7 que contiene dos lazos, se halla la función de transferencia del lazo interior por

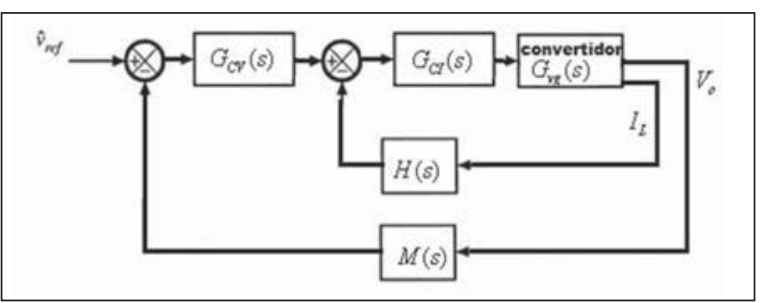

Figura 7. Esquema del convertido en lazo cerrado.

Fuente: elaboración propia

medio de reducción de bloques y posteriormente se realiza un procedimiento similar con el lazo exterior. Esta última función de transferencia representa el comportamiento del sistema en lazo cerrado. A continuación se analizan los diagramas de bode para observar su comportamiento en frecuencia.

El lazo de retroalimentación puede ser usado para disminuir la influencia del voltaje del generador y la corriente de carga sobre el voltaje de salida $V o(s)$, por tal motivo, para el análisis del lazo cerrado se utiliza la función de transferencia del voltaje de salida con respecto al voltaje de entrada como la función de transferencia del convertidor de potencia.

Una vez realizada la reducción en diagrama de bloques, se obtiene la función de transferencia mostrada en la ecuación (31) donde $R$ es la resistencia de carga, $L$ es la inductancia, $R_{l}$ resistencia de la inductancia, $R_{O N}$ resistencia de encendido del MOSFET, $R_{d}$ resistencia interna del diodo, $C$ es la capacitancia de salida y $V_{S}$ voltaje de alimentación del convertidor Boost hallados en las secciones anteriores (sección 3).

$$
G_{\text {Tlowe }}(s)=\frac{\left[\frac{s}{16800}+1\right] \alpha_{3}}{\left[\begin{array}{l}
\frac{\alpha_{5}}{235000} s^{4}+\left(\frac{\alpha_{5}}{(235000 * \mathrm{RC})}+\frac{\alpha_{1} \alpha_{5}}{(235000 * \mathrm{~L})}+\alpha_{5}\right) \mathrm{s}^{3}+\left(\frac{\alpha_{5} \alpha_{1}}{(235000 * \mathrm{RLC})}+\frac{\alpha_{5} \mathrm{D}^{\prime}}{(235000 \mathrm{LC})}+\frac{\alpha_{5} \alpha_{1}}{\mathrm{~L}}+\frac{\alpha_{5}}{(\mathrm{RC})}+\frac{\alpha_{6}}{(16800 * 6262)}\right) \mathrm{s}^{2} \\
+\left(\frac{\alpha_{1} \alpha_{5}}{(\mathrm{RLC})}+\frac{\mathrm{D}^{\prime} \alpha_{5}}{(\mathrm{LC})}+\frac{\alpha_{6}}{16800}+\frac{\alpha_{6}}{6262}\right) \mathrm{s}+\left(\alpha_{6}+\alpha_{4}\right)
\end{array}\right]}
$$




\section{investigación}

Donde se realizaron las sustituciones planteadas en la ecuación (31).

$$
\begin{aligned}
& \alpha_{1}=\left(R_{l}+R_{O N} D+R_{d} D^{\prime}\right) \\
& \alpha_{3}=\frac{\mathrm{D}^{\prime}}{(\mathrm{LC})} *\left(\mathrm{CRV}_{s}\right) * 6.0949 \mathrm{e}^{6} \\
& \alpha_{4}=\frac{\mathrm{D}^{\prime}}{(\mathrm{LC})}\left(\mathrm{CRV}_{s}\right) * 2.8992 \\
& \alpha_{5}=(\mathrm{CR}) * 80 \\
& \alpha_{6}=4232420.9 * \frac{\mathrm{D}^{\prime}}{\left(\mathrm{L}^{*} \mathrm{C}\right)}
\end{aligned}
$$

Para analizar la función de transferencia de la ecuación (30), se grafican los diagramas de bode con los cuales se observa una frecuencia de corte de $162 \mathrm{rad} / \mathrm{seg}$ y un margen de fase positivo de $120 \mathrm{deg}$. Como se observa en la figura 8.

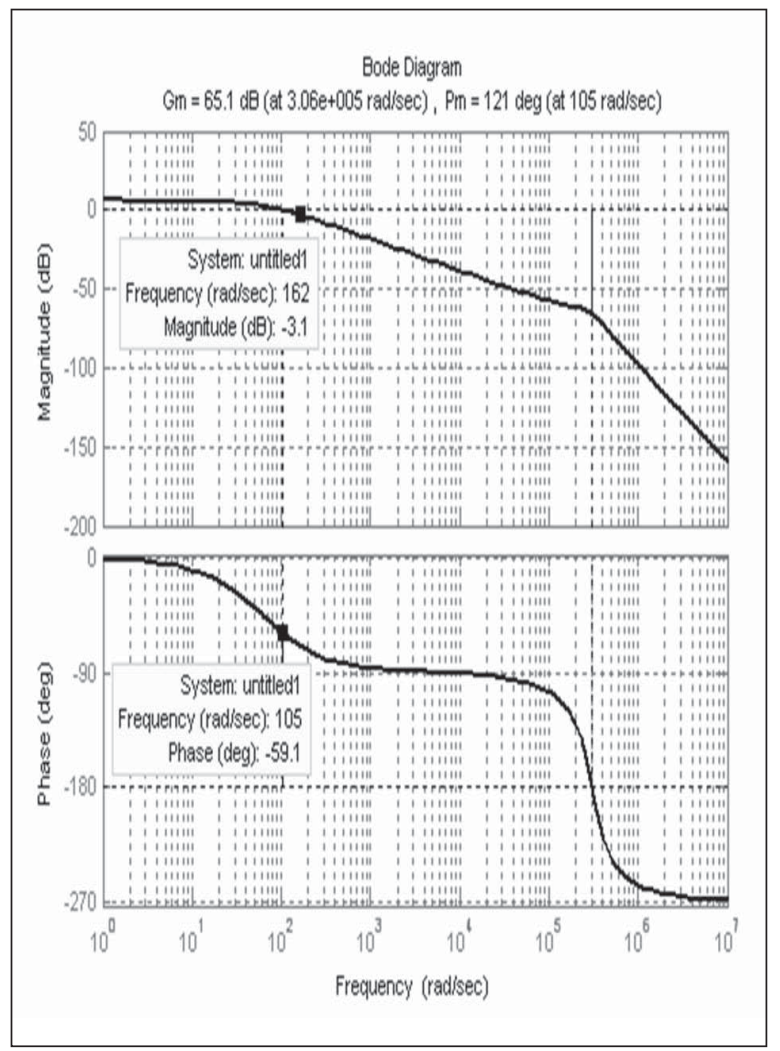

Figura 8. Diagrama de bode del convertidor de potencia Boost controlado para corregir el factor de potencia.

Fuente: elaboración propia

\section{RESULTADOS}

\subsection{Simulación del modelo matemático del convertidor Boost}

Por medio de la herramienta S-Fuction [11] se observa el comportamiento del modelo matemático en simulink/matlab. En la figura 9 se observa el voltaje en la carga que presentará el convertidor Boost.

En la figura 10 se observa la forma de onda de la corriente que circula a través de la bobina, la cual será una señal totalmente rectificada.

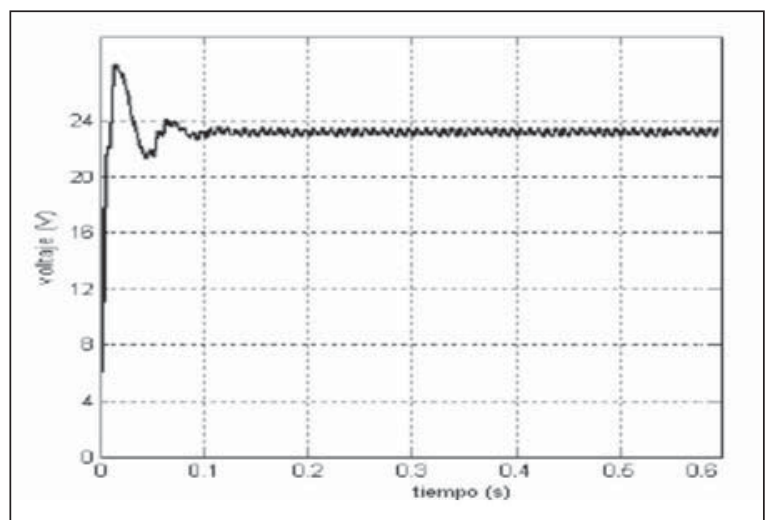

Figura 9. Gráfica de voltaje en la carga con el modelo matemático (5V/div).

Fuente: elaboración propia

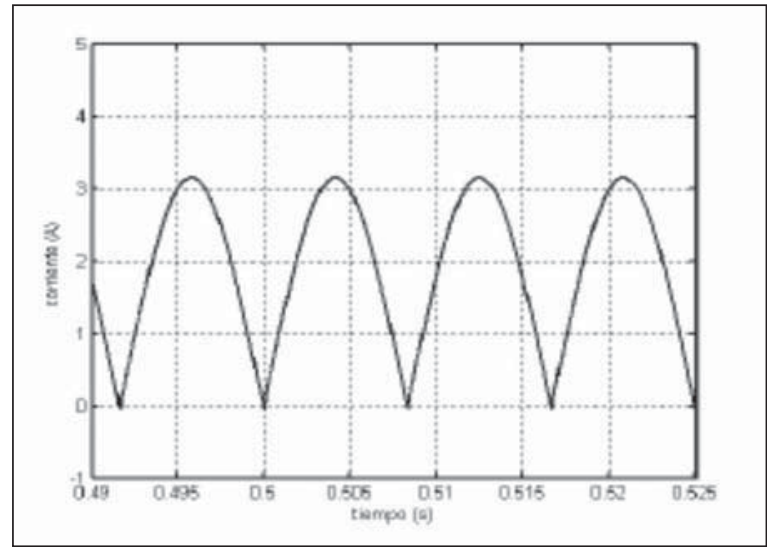

Figura 10. Gráfica de la corriente en la bobina con el modelo matemático (1 $\mathrm{A} / \mathrm{div})$.

Fuente: elaboración propia 


\subsection{Simulación del modelo conmutado del convertidor Boost}

En la figura 11 se observa la forma de onda del voltaje presente en la carga y la respuesta del sistema ante variaciones de carga de $250 \Omega$ a $25 \Omega$.

En la figura 12 se observa la forma de onda de la corriente del generador y su respuesta ante variaciones de carga de $250 \Omega$ a $25 \Omega$, la cual es fundamental para analizar la corrección del factor de potencia.

En la tabla 1 se presenta la comparación de la simulación del convertidor con y sin control, se puede apreciar la mejora en la regulación de voltaje, distorsión armónica y factor de potencia cuando el convertidor está controlado, teniendo en cuenta un ciclo de trabajo de 50\% para el convertidor sin control.

\subsection{Resultados experimentales}

Los resultados obtenidos al implementar el convertidor se observan en las figuras 13 y 14. En la

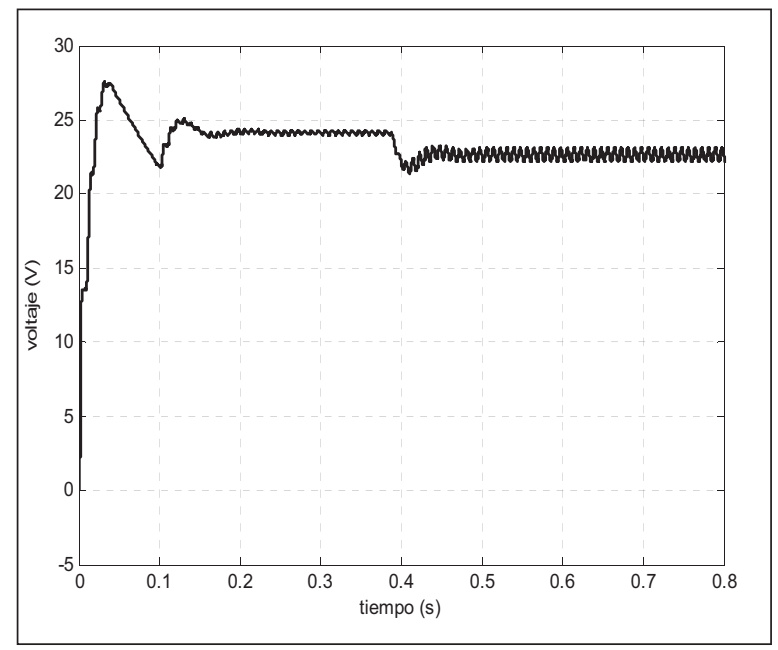

Figura 11. Gráfica de voltaje en la carga con el modelo conmutado (5V/div).

Fuente: elaboración propia
Tabla 1. Comparación de las respuestas del convertidor tipo boost con control y sin control

\begin{tabular}{|l|c|c|}
\hline & $\begin{array}{c}\text { Convertidor } \\
\text { Boost } \\
\text { controlado }\end{array}$ & $\begin{array}{c}\text { Convertidor } \\
\text { Boost } \\
\text { sin control }\end{array}$ \\
\hline $\begin{array}{l}\text { \% de regulación } \\
\text { de voltaje }\end{array}$ & $3,6 \%$ & $19,9 \%$ \\
\hline $\begin{array}{l}\% \text { de distorsión } \\
\text { armónica }\end{array}$ & $11 \%$ & $78 \%$ \\
\hline Factor de potencia & 0,995 & 0,82 \\
\hline
\end{tabular}

Fuente: elaboración propia

figura 13 se muestra el oscilograma de la corriente de entrada del prototipo en lazo abierto y en la figura 14 se muestra el oscilograma de la misma señal cuando se cierra el lazo de control, se puede apreciar el efecto de corrección de su forma de onda y la disminución en magnitud y cantidad del contenido armónico.

En la figura 15 se muestra el oscilograma del voltaje de salida sin carga y a plena carga, donde se puede resaltar el incremento del rizado de un $1.25 \%$ sin carga, a un $3.8 \%$ a plena carga y la presencia de ruido inherente a las conmutaciones del convertidor.

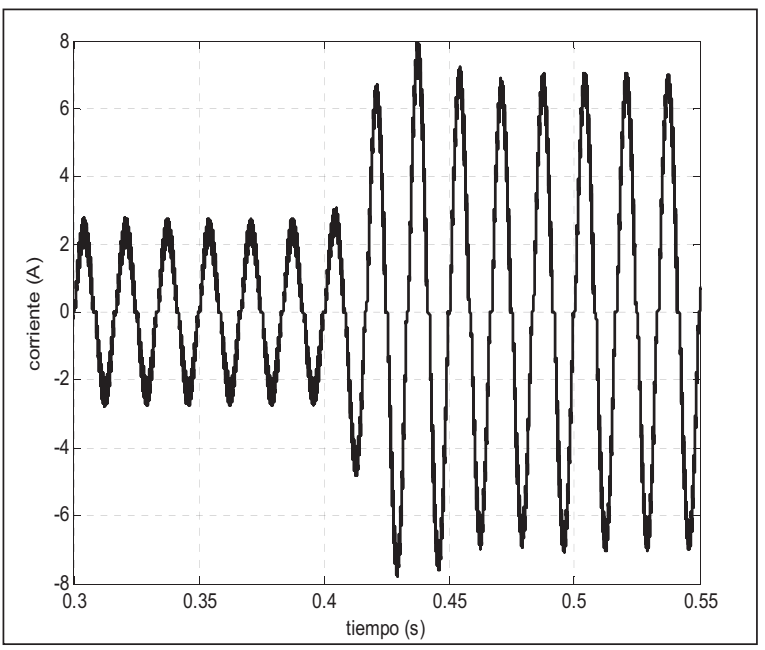

Figura 12. Gráfica de la corriente en el generador con el modelo conmutado (2A/div).

Fuente: elaboración propia 


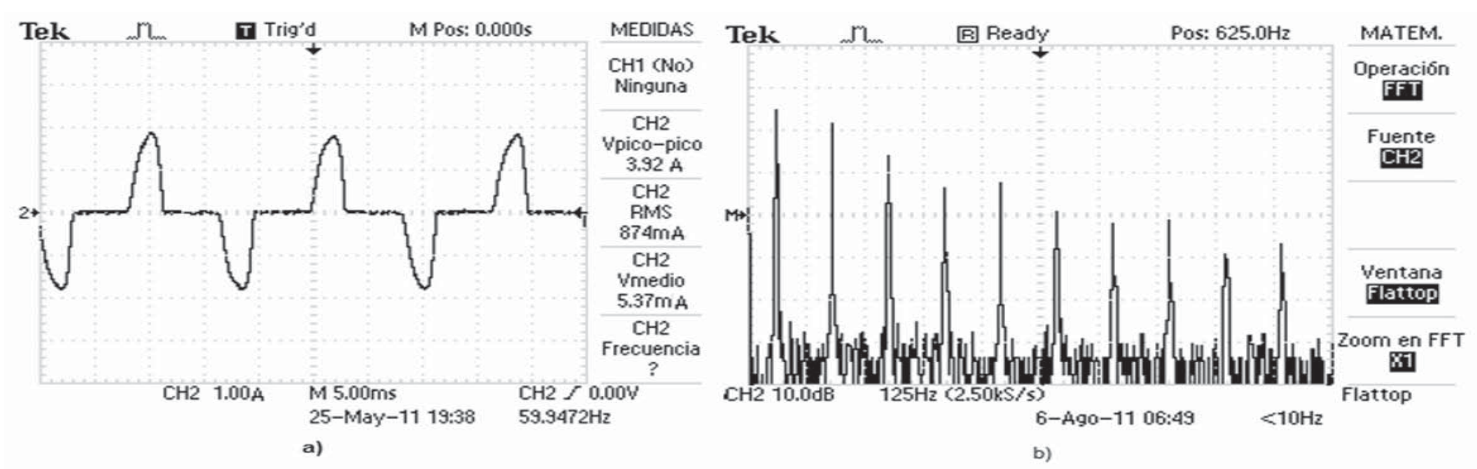

Figura 13. Oscilograma de a) señal de corriente de entrada en lazo abierto. b) FFT de la señal en lazo abierto. Fuente: elaboración propia

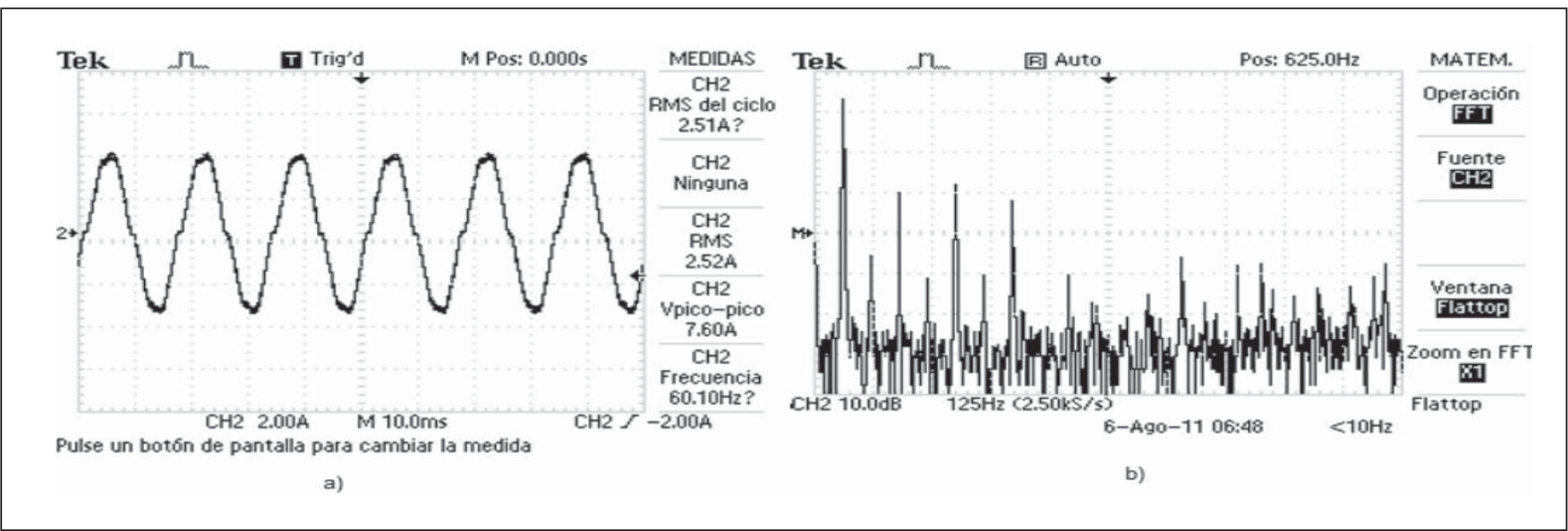

Figura 14. Oscilograma de a) señal de corriente de entrada con control. b) FFT de la señal con control. Fuente: elaboración propia

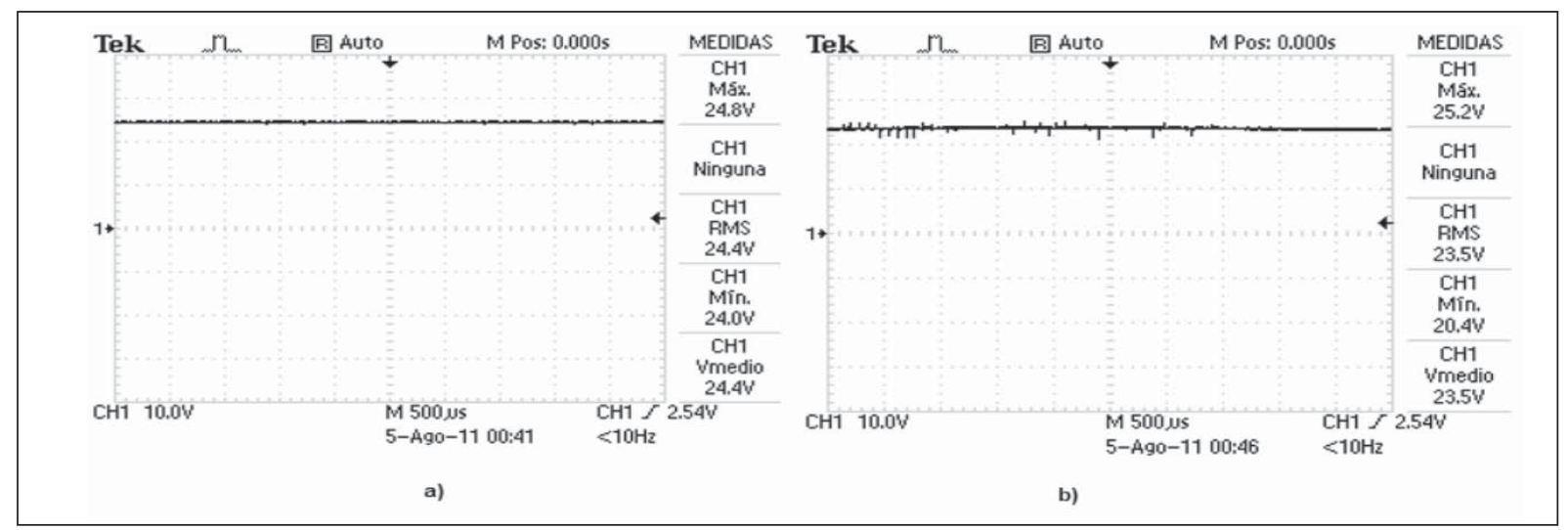

Figura 15. Oscilograma del voltaje de salida a) sin carga b) plena carga.

Fuente: elaboración propia 


\section{investigación}

\section{CONCLUSIÓN}

Se desarrolló un rectificador con corrección de factor de potencia utilizando la topología del convertidor Boost, controlado por compensadores PI, con un excelente desempeño, ya que se disminuyó la distorsión armónica en un 67\%, con una potencia de salida de $24 \mathrm{~W}$, sus parámetros de diseño fueron comprobados mediante simulación haciendo uso del programa simulink/ Matlab obteniendo una eficiencia de $81 \%$ y con los resultados experimentales se obtuvo una eficiencia de $71 \%$.

\section{FINANCIAMIENTO}

El proyecto fue financiado por los autores y comprobado por medio de los instrumentos de medida presentes en el laboratorio de electrónica de la UPTC. Este proyecto fue avalado como proyecto de grado en la misma universidad.

\section{REFERENCIAS}

[1] Suornitec, [Online] [Cited: 02 25, 2011.] Available: www.suornitec.com/suornitec/ armonicos.htm.

[2] D. U. Campos Delgado, Experimentos en teoría de control. Convertidores DC-DC. s.1. : Facultad de Ciencias (UASLP), 2001.

[3] M. Gonzales Trigo y R. Massano, Factor de potencia. s.l. : Facultad de ingenieria, 2009.

[4] N. Singh, Brij, et al, Review of single-phase improved power quality ac-dc converters ieee transactions on industrial electronics, Vol. 50. Canada : IEEE, 2003.

[5] F. Tahami, H. B. Gholami and H. M. Ahmadian, Modeling of the Boost Power Factor Correction. Tehran: IEEE, 2008.

[6] Zhou, Chen, Ridley, B. Raymond and Lee, C. Fred, Design and analysis of a hysteretic boost. Virginia : IEEE, 2002.
[7] R. W. Erickson, Fundamentals of power electronics. Usa: Kluwer academic publishers, 2001.

[8] H.Y. Kanaan, K. Sauriol and A.L. Haddad, Small-signal modelling and linear control of a high efficiency dual boost single-phase, Vol. 2. Marruecos : ESIB, 2009.

[9] H. Y. Kanaan and A. Hayek, Small-Signal Averaged Model and Carrier-Based, Vol. 30. Canada: IEEE, 2008.

[10] N. Mohan, Power electronics and drives. Minneapolis: minpere, 2003.

[11] Matworks. Matlab R2009a. [Online] 2009. [Cited: 02 10, 2011] Available: www.matworks.com/help/toolbox/physmod/powersys.

[12] S. Dui-jia, D. J. Adams and M. T. Leon, Comparative study of power factor correction converters for single phase half-bridge inverters. s.1. : PESC, 2001. 